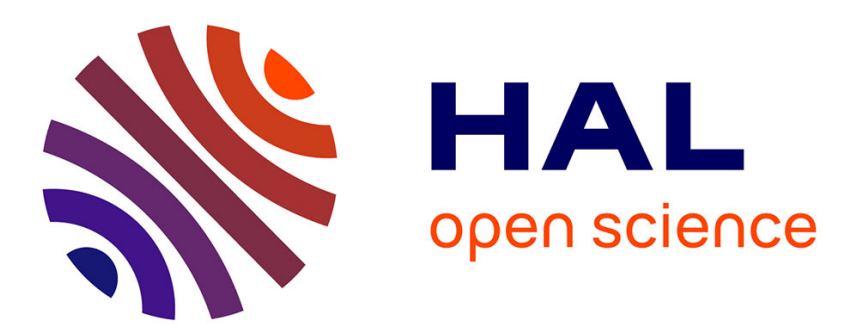

\title{
Spectral transmittance model for stacks of transparencies printed with halftone colors
}

Jacques Machizaud, Mathieu Hébert

\section{To cite this version:}

Jacques Machizaud, Mathieu Hébert. Spectral transmittance model for stacks of transparencies printed with halftone colors. Color Imaging XVII: Displaying, Processing, Hardcopy, and Applications, Feb 2012, Burlingame (CA), United States. pp.829212, 10.1117/12.905552 . hal-00858739

\section{HAL Id: hal-00858739 \\ https://hal.science/hal-00858739}

Submitted on 6 Sep 2013

HAL is a multi-disciplinary open access archive for the deposit and dissemination of scientific research documents, whether they are published or not. The documents may come from teaching and research institutions in France or abroad, or from public or private research centers.
L'archive ouverte pluridisciplinaire HAL, est destinée au dépôt et à la diffusion de documents scientifiques de niveau recherche, publiés ou non, émanant des établissements d'enseignement et de recherche français ou étrangers, des laboratoires publics ou privés. 


\title{
Spectral transmittance model for stacks of transparencies printed with halftone colors
}

\author{
Jacques Machizaud ${ }^{\mathrm{a}}$, Mathieu Hébert ${ }^{\mathrm{a}}$ \\ ${ }^{a}$ Université de Lyon, Université Jean-Monnet de Saint-Etienne; CNRS, UMR 5516, Laboratoire \\ Hubert Curien, F-42000, Saint-Etienne, France.
}

\begin{abstract}
The present work investigates the transmission of light through stacks of halftone printed transparencies. We propose a spectral transmittance model describing the multiple reflections of light between the transparencies, whose individual reflectance and transmittance have themselves been obtained by a prediction model. The model for single printed transparency involves the multiple reflections of light between the interfaces as well as the orientation-dependent attenuations of light within the plastic and ink layers. A procedure enables converting the nominal ink surface coverages into effective ones by taking into account the spreading of the inks. Calibration of the model is based on printing a small number of color patches on one transparency and measuring their spectral transmittance. Regarding the stacks of transparencies, an experimental test carried out with inkjet printed samples shows good agreement between predictions and measurements for stacks of two, three and four transparencies. Stochastic halftones are used in order to avoid the apparition of moiré patterns when superposing the halftones. By inversion of the model, we are able to determine the halftone colors to print on each transparency in order to obtain by superposition one targeted color. An original application of this, called "color matching", consists in producing one color of stack from various combinations of colors on the transparencies. The prediction accuracy of the proposed model guarantees the good visual uniformity of the resulting colored area.
\end{abstract}

Keywords: Color reproduction, spectral transmittance prediction model, multilayer, halftone color, color matching

\section{INTRODUCTION}

Thanks to recent spectral prediction models developed in the color reproduction domain, especially the extension of classical spectral reflectance models to the spectral transmittance ${ }^{1}$ and the consideration of the ink spreading in the calibration $\operatorname{step}^{2}$, we have now good experience to predict accurately the color rendering of halftone prints in reflectance and transmittance mode. The study of stacks of uniformly colored nonscattering sheets was also a first step towards the prediction of the spectral reflectance and transmittance of multilayer specimens ${ }^{3}$. In the present study, we propose to combine both models for halftone prints and for stacks of nonscattering sheets in order to predict the spectral transmittance of stacks of nonscattering transparencies printed in halftone. The model for stacks of nonscattering sheets was inspired of the Kubelka compositional equations ${ }^{4}$, which have been adapted to the case of nonscattering multilayers where the multiple reflection process taking place between the interfaces depends on the orientation of the incident light and on the refractive index of the different layers. In the present case, the color of the plastic layers is not uniform as in the previous study but composed of a mosaic of colored areas, characteristic of the halftone colors. Predicting the spectral reflectances and transmittances of the printed transparencies before combining them in order to obtain the spectral transmittance of the stack is the main contribution of the present work.

Regarding the color of one transparency, we develop a reflectance and transmittance model which combines the classical reflectance and transmittance model for a thick slab with parallel surfaces and the Yule-Nielsen enhanced spectral Neugebauer (YNSN) model which renders the non-linear relationship between the spectral properties of the halftone and the surface coverages of the colored areas in the halftone. This model, presented in Section 2, is calibrated from spectral reflectance and transmittance measurements of a small number of printed colors, performed in the normal direction in 
respect to the transparency. Once calibrated, the model should be able to predict the spectral reflectance and transmittance for any halftone and any illumination geometry, e.g. the radiance in one direction or a Lambertian irradiance over the hemisphere.

Once the printed transparencies are stacked together, a multiple reflection process of light occurs between them. We model it by describing flux transfers between the light sources, the transparencies and the detector. We obtain analytical expressions for the transmittance of the specimens, which are functions of the intrinsic parameters of the stacked transparencies. A difficulty of this model compared to previous works comes from the fact that two superposed halftones generated by classical periodical dithering produce moirés ${ }^{5}$. In order to prevent them, we generate the halftones by the stochastic method detailed in Refs. [6] and [7].

This paper is structured as follows. First, we briefly recall radiometric and optical concepts used in our model. Then, we explain how to predict the spectral reflectance and transmittance of one printed transparency (Section 3) and of a stack of printed transparencies (Section 4). The experimental testing is analyzed in Section 5 and an original application of the model is described in Section 6. Finally, Section 7 draws the conclusions.

\section{RADIOMETRIC AND OPTICAL BACKGROUNDS}

The spectral prediction model that we proposed for stacks of printed transparencies is based on a description of the propagation of light into several layers combining the reflections and refractions of light by the interfaces and the spectral attenuations of light by the inks. The amounts of light concerned by these elementary phenomena are assessed thanks to radiometric concepts ${ }^{8}$.

In the present case, the inks and the plastic transparencies are considered as non-scattering layers. In a non-scattering medium, the light is attenuated according to Beer's law'. If we denote as $t$ the attenuation applied to a light ray crossing a purely absorbing layer in the normal direction in respect to the layer (normal transmittance), the attenuation becomes $t^{1 / \cos \theta}$ as the ray forms an angle $\theta$ to the normal of the layer.

The refractive indices of the plastic and the inks are fairly close each other. Here we take $\mathrm{n}_{1}=1.5$ for their common value. At their interface with air, whose refractive index $\mathrm{n}_{0}$ is close enough to unity, light undergoes reflection and refraction. Assuming that the interfaces are flat, reflection and refraction satisfy Snell's laws and the proportions of directional fluxes being reflected and transmitted are given by the Fresnel Formulae'. We denote as $R_{j k}\left(\theta_{j}\right)$ and $T_{j k}\left(\theta_{j}\right)$ the reflectivity, respectively the transmittivity of the interface when natural incident light comes at angle $\theta_{j}$ from medium $j$. The interface has the same reflectivity when the pencil comes from medium $k$ at the angle $\theta_{k}=\arcsin \left(n_{j} \sin \theta_{j} / n_{k}\right)$ being related to $\theta_{j}$ according to Snell's refraction law ${ }^{10}$ :

$$
R_{k j}\left(\theta_{k}\right)=R_{j k}\left(\theta_{j}\right)
$$

Since the energy is conserved at the interface, the Fresnel transmittivity $T_{j k}\left(\theta_{j}\right)$ is related to the Fresnel reflectivity by:

$$
T_{j k}\left(\theta_{j}\right)=1-R_{j k}\left(\theta_{j}\right)
$$

The reciprocity property stated by Eq. (1) applies to transmittivity.

\section{STACKS OF UNPRINTED TRANSPARENCIES}

Before presenting the model for transparencies printed with halftone colors, let us first recall the main lines of the model previously proposed for stacks of unprinted nonscattering slabs ${ }^{3}$. The slabs are made of the same material with refractive index $n_{1}$ and are all separated by air slices. This is the case if no other medium is used to paste the slabs. 


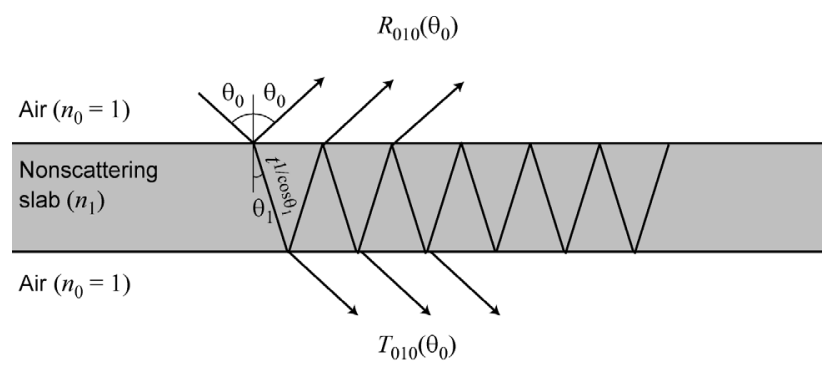

Figure 1: Reflection and transmission of light by a nonscattering slab.

The reflectance and transmittance of one slab of refractive index $n_{1}$ and normal transmittance $t$ are derived by describing the multiple reflection process of light between the surfaces and taking into account the orientation-dependent attenuation of the light components in the slab (Figure 1). Since the surfaces are parallel, the orientation of light in the slab remains constant after each reflection. It is denoted by the angle $\theta_{1}$, related to the incident angle $\theta_{0}$ in air by Snell's law, i.e. $\theta_{1}=\arcsin \left(n_{0} \sin \theta_{0} / n_{1}\right)$. According to reciprocity formula (1), all reflections concern a fraction $R_{01}\left(\theta_{0}\right)=R_{10}\left(\theta_{1}\right)$ of light and all transmissions concern a fraction $T_{01}\left(\theta_{0}\right)=T_{10}\left(\theta_{1}\right)$ of light. By following the multiple reflections process represented in Figure 1 and summing up the different components exiting at each side of the slab, one obtains geometrical series expressing the reflectance and transmittance of the slab ${ }^{3}$. Once reduced, the geometrical series provide the following reflectance and transmittance formulae:

$$
R_{010}\left(\theta_{0}\right)=R_{01}\left(\theta_{0}\right)+\frac{T_{01}^{2}\left(\theta_{0}\right) R_{01}\left(\theta_{0}\right) t^{2 / \cos \theta_{1}}}{1-R_{01}^{2}\left(\theta_{0}\right) t^{2 / \cos \theta_{1}}},
$$

respectively,

$$
T_{010}\left(\theta_{0}\right)=\frac{T_{01}^{2}\left(\theta_{1}\right) t^{1 / \cos \theta_{1}}}{1-R_{01}^{2}\left(\theta_{0}\right) t^{2 / \cos \theta_{1}}}
$$

Equations (3) and (4) are valid for either $p$ - or s-polarization of light, by considering the corresponding Fresnel formulas for the reflectivities and transmittivities of the interfaces. With natural incident light, the reflectance is the average of the $p$-polarization and s-polarization reflectance contributions. However, we observed that with typical transparencies used for inkjet printers, it does not make an appreciable difference to use the Fresnel reflectivities and transmittivities defined for natural light, i.e. to average the Fresnel coefficients before summing rather than the opposite, which provides simpler equations.

At normal incidence, the reflectivity $R_{01}$ of the interfaces becomes $\left(n_{1}-1\right)^{2} /\left(n_{1}+1\right)^{2}$. The transmittance formula (4) at $\theta_{0}=0$ depends only on the refractive index $n_{1}$ and on $t(\lambda)$. It therefore enables deducing $t(\lambda)$ as soon as the refractive index of the transparency and its spectral transmittance $T_{010}(0, \lambda)$ in the normal direction are known:

$$
t(\lambda)=\frac{\sqrt{64 n_{1}^{4}+\left(1-n_{1}^{2}\right)^{4} T_{010}^{2}(0, \lambda)}-8 n_{1}^{2}}{\left(1-n_{1}\right)^{4} T_{010}(0, \lambda)}
$$

When the transparency is coated with a uniform layer of ink, one obtains a new non-scattering slab whose normal transmittance $t_{i}(\lambda)$ is given by similar formula as formula (5), with $T_{010}(0, \lambda)$ denoting the normal transmittance of the inked transparency measured at normal incidence. Once the normal transmittance has been determined, the reflectance and transmittance of the transparency can be determined for every incident angle according to Eqs. (3) and (4) respectively. 
Let us now consider a stack of several transparencies with different normal transmittances. All the transparencies are separated by air in which light is oriented by the same angle $\theta_{0}$ as in the surrounding air. We can assume that each transparency has the same reflectance at its top and bottom sides. We denote as $R_{i}\left(\theta_{0}\right)$ and $T_{i}\left(\theta_{0}\right)$ the reflectance of the $i$ th transparency in the stack, numbered from top to bottom.

Let us first consider two superposed transparencies, labeled 1 on the top side and 2 on the bottom side. Their reflectance at the top side is ${ }^{4}$

$$
R_{12}\left(\theta_{0}\right)=R_{1}\left(\theta_{0}\right)+\frac{T_{1}^{2}\left(\theta_{0}\right) R_{2}\left(\theta_{0}\right)}{1-R_{1}\left(\theta_{0}\right) R_{2}\left(\theta_{0}\right)}
$$

their reflectance at the bottom side is

$$
R_{21}\left(\theta_{0}\right)=R_{2}\left(\theta_{0}\right)+\frac{T_{2}^{2}\left(\theta_{0}\right) R_{1}\left(\theta_{0}\right)}{1-R_{1}\left(\theta_{0}\right) R_{2}\left(\theta_{0}\right)}
$$

and their transmittance is

$$
T_{12}\left(\theta_{0}\right)=\frac{T_{1}\left(\theta_{0}\right) T_{2}\left(\theta_{0}\right)}{1-R_{1}\left(\theta_{0}\right) R_{2}\left(\theta_{0}\right)}
$$

Note that the reflectances of the stack at the top and bottom sides are different.

When three transparencies are superposed, we first consider the two first ones whose reflectance and transmittance are given by the equations above, and then consider them superposed to the third transparency. The reflectance and transmittance of the stack is given by similar equations as Eqs. (6) to (8) by replacing index 1 with index 12 and index 2 with index 3. More generally, knowing the reflectances of $i$ superposed transparencies at the top and bottom sides as well as their transmittance, the reflectance of these $i$ transparencies plus a $(i+1)$ th one, denoted by index $j$ for simpler notations, is

$$
R_{1 . . i j}\left(\theta_{0}\right)=R_{1 . . i}\left(\theta_{0}\right)+\frac{T_{1 . . i}^{2}\left(\theta_{0}\right) R_{j}\left(\theta_{0}\right)}{1-R_{i . .1}\left(\theta_{0}\right) R_{j}\left(\theta_{0}\right)},
$$

their reflectance at the bottom side is

$$
R_{j i .11}\left(\theta_{0}\right)=R_{j}\left(\theta_{0}\right)+\frac{T_{j}^{2}\left(\theta_{0}\right) R_{i .1}\left(\theta_{0}\right)}{1-R_{i .1}\left(\theta_{0}\right) R_{j}\left(\theta_{0}\right)},
$$

and their transmittance is

$$
T_{1 . . i j}\left(\theta_{0}\right)=\frac{T_{1 . . i}\left(\theta_{0}\right) T_{j}\left(\theta_{0}\right)}{1-R_{i . .1}\left(\theta_{0}\right) R_{j}\left(\theta_{0}\right)}
$$

For a stack of $N$ transparencies, Eqs. (9) to (11) may be used iteratively by considering first the two first transparencies, then the three first ones, the four first ones and so on. The obtained reflectance and transmittance expressions for the stack have analytical expressions as functions of the incident angle $\theta_{0}$, the refractive index of the transparencies and their respective normal transmittances.

\section{STACKS OF TRANSPARENCIES PRINTED WITH HALFTONE COLORS}

We now extend the model presented in the previous section to the case where the transparencies are printed with halftone colors. A halftone is a mosaic of colored areas from partial overlap of the ink dot screens. The areas with no ink, those with a single ink layer, and those with two or three superposed ink layers are each one considered as a distinct 
"colorant", also called "Neugebauer primary". For three primary inks (e.g. cyan, magenta and yellow), one obtains a set of eight colorants: white (no ink), cyan alone, magenta alone, yellow alone, red (magenta \& yellow), green (cyan \& yellow), blue (cyan \& magenta) and black (cyan \& magenta \& yellow). We denote as $R^{(k)}\left(\theta_{0}\right)$ and $T^{(k)}\left(\theta_{0}\right)$ the reflectance, respectively the transmittance of a transparency coated on its whole surface by the colorant $k$. Most of the time, these ones are measured at normal incidence, i.e. for $\theta_{0}=0$. If the reflectance or transmittance at another incident angle is needed, one may deduce the normal transmittance of the inked transparency according to Eq. (5), then predict the reflectance of transmittance at the desired angle from Eq. (3), or accordingly Eq. (4).

In classical clustered dot or error diffusion prints, the fractional area $a_{k}$ occupied by each colorant can be deduced from the surface coverages $x_{c}, x_{m}$ and $x_{y}$ of the three primary inks according to Demichel's equations ${ }^{12}$

$$
\begin{aligned}
& a_{w}=\left(1-x_{c}\right)\left(1-x_{m}\right)\left(1-x_{y}\right) \\
& a_{c}=x_{c}\left(1-x_{m}\right)\left(1-x_{y}\right) \\
& a_{\bar{m}}=\left(1 \quad x_{c}\right) x_{m}\left(1 \quad x_{y}\right) \\
& a_{y}=\left(1-x_{c}\right)\left(1-x_{m}\right) x_{y} \\
& a_{m+y}=\left(1-x_{c}\right) x_{m} x_{y} \\
& a_{c+y}=x_{c}\left(1-x_{m}\right) x_{y} \\
& a_{c+m}=x_{c} x_{m}\left(1-x_{y}\right) \\
& a_{c+m+y}=x_{c} x_{m} x_{y}
\end{aligned}
$$

For a transparency coated by a halftone color, we may assume in first approach that each colorant area reflects light as when the colorant covers all the transparency, i.e. the reflectance of the halftone is the sum of the reflectances $R^{(k)}\left(\theta_{0}\right)$ weighted by the surface coverages $a_{k}$ :

$$
R\left(\theta_{0}\right)=\sum_{k=1}^{8} a_{k} R^{(k)}\left(\theta_{0}\right)
$$

However, this model, known as Spectral Neugebauer model, provides poor predictions because it does not account for the transition of light from one colorant to another during the multiple reflection process. These transitions, well known on paper due to the scattering of light in the paper bulk, lead to a similar effect as the Yule-Nielsen effect. We take it into account by applying to Eq. (13) the Yule-Nielsen transform, i.e.:

$$
R\left(\theta_{0}\right)=\left[\sum_{k=1}^{8} a_{k}\left(R^{(k)}\left(\theta_{0}\right)\right)^{1 / n}\right]^{n}
$$

where $n$ is a number to be fitted in the calibration step. The experience shows that the reflectance of a single transparency is the same on its two sides.

For the same reason, we apply the Yule-Nielsen transform to the Spectral Neugebauer equation in transmittance mode:

$$
T\left(\theta_{0}\right)=\left[\sum_{k=1}^{8} a_{k}\left(T^{(k)}\left(\theta_{0}\right)\right)^{1 / n}\right]^{n}
$$

Due to the spreading of the inks on the surface of the transparency, the surface coverages are different to the expected ones. In order to determine the effective surface coverages, ink spreading curves are computed from the measurement of specific printed halftones, where one ink is halftoned at several nominal surface coverages and the other inks are printed at nominal surface coverages 0 or 1 , and so on for each ink. In each of these specific halftones, we have two colorants $u$ 
and $v$, with respective surface coverage $a$ and $(1-a)$. According to Eq. (15), the spectral transmittance $T_{u / v}(a, \lambda)$ of the transparency printed with this halftone is

$$
T_{u / v}(a, \lambda)=\left[(1-a) T_{u}^{1 / n}(\lambda)+a T_{v}^{1 / n}(\lambda)\right]^{n}
$$

We determine the effective surface coverage $a_{u / v}$ by fitting the parameter $a$ which minimizes the sum of squared differences between the spectral transmittance $T_{u / v}(a, \lambda)$ and the measured spectral transmittance $Q_{u / v}(\lambda)$

$$
a_{u / v}=\underset{a}{\operatorname{atg} \min }\left[\sum_{\lambda} T_{u / v}(a,) Q_{u / v}()\right]
$$

Once, the effective surface coverages $a_{u / v}$ are calculated for all the specific halftones, we establish the ink spreading curves $f_{u / v}(a)$ giving the effective surface coverage from the nominal surface coverage by linear interpolation as explained in Ref. [4].

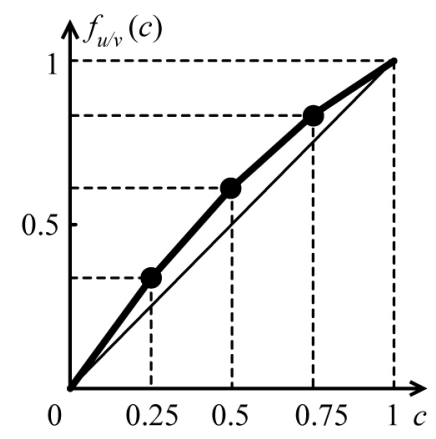

Figure 2. Example of ink spreading curve, giving the effective surface coverage of ink $u$ when superposed on colorant $v$ as a function of the nominal surface coverage $c$.

Let us now consider a three-ink halftone where the cyan, magenta and yellow inks have the respective nominal surface coverages $x_{c}, x_{m}$, and $x_{y}$. These nominal ink surface coverages are converted into effective ink surface coverages $x_{c}^{\prime}, x_{m}^{\prime}$ and $x_{y}^{\prime}$ which account for the superposition-dependent ink spreading. Effective surface coverages of an ink halftone are obtained by a weighted average of the ink spreading curves. The weights are expressed by the surface coverages of the respective colorants on which the ink halftone is superposed. For example, the weight of the ink spreading curve $f_{c}$ (cyan halftone over white colorant) is $\left(1-x_{m}^{\prime}\right)\left(1-x_{y}^{\prime}\right)$. In the case of three halftoned inks, effective surface coverages are obtained by performing a few iterations with the following equations:

$$
\begin{aligned}
& x_{c}^{\prime}=\left(1-x_{m}^{\prime}\right)\left(1-x_{y}^{\prime}\right) f_{c}\left(x_{c}\right)+x_{m}^{\prime}\left(1-x_{y}^{\prime}\right) f_{c / m}\left(x_{c}\right)+\left(1-x_{m}^{\prime}\right) x_{y}^{\prime} f_{c / y}\left(x_{c}\right)+x_{m}^{\prime} x_{y}^{\prime} f_{c / m+y}\left(x_{c}\right) \\
& x_{m}^{\prime}=\left(1-x_{c}^{\prime}\right)\left(1-x_{y}^{\prime}\right) f_{m}\left(x_{m}\right)+x_{c}^{\prime}\left(1-x_{y}^{\prime}\right) f_{m / c}\left(x_{m}\right)+\left(1-x_{c}^{\prime}\right) x_{y}^{\prime} f_{m / y}\left(x_{m}\right)+x_{c}^{\prime} x_{y}^{\prime} f_{m / c+y}\left(x_{m}\right) \\
& x_{y}^{\prime}=\left(1-x_{c}^{\prime}\right)\left(1-x_{m}^{\prime}\right) f_{y}\left(x_{y}\right)+x_{c}^{\prime}\left(1-x_{m}^{\prime}\right) f_{y / c}\left(x_{y}\right)+\left(1-x_{c}^{\prime}\right) x_{m}^{\prime} f_{y / m}\left(x_{y}\right)+x_{c}^{\prime} x_{m}^{\prime} f_{y / c+m}\left(x_{y}\right)
\end{aligned}
$$

For the first iteration, $x_{c}^{\prime}=x_{c}, x_{m}^{\prime}=x_{m}$ and $x_{y}^{\prime}=x_{y}$ are taken as initial values on the right side of the equations. The obtained values of $c, m$ and $y$ are then inserted again into the right side of the equations, which gives new values of $x_{c}, x_{m}$, and $x_{y}$ and so on, until the values of $x_{c}, x_{m}, x_{y}$ stabilize. The effective surface coverages of the colorants are calculated by plugging the obtained values for $x_{c}, x_{m}$, and $x_{y}$ into the Demichel equations (12).

When several transparencies are superposed, we simply follow the iterative procedure presented in Section 3 to obtain the reflectances of the stack at the upper and the lower sides as well as the transmittance. Each transparency is characterized by its reflectance and transmittance at the considered angle $\theta_{0}$ given by Eqs. (14) and (15), respectively. These latter depend on the reflectances $R^{(k)}$, respectively on the transamittances $T^{(k)}$ of the solid colorant patches printed 
on the transparency, which are either determined by measurement at the angle $\theta_{0}$ or predicted according to Eq. (3), respectively Eq. (4), from the normal transmittance $t^{(k)}$ deduced from measurement at normal incidence according to Eq. (5). Assuming that all transparencies in the stack are identical and printed with the same inks, the same printing system and the same halftoning method, the same model can be used to predict all the individual reflectances $R_{i}$ and transmittances $T_{i}$.

\section{EXPERIMENTAL TESTING}

We tested the model on stacks of two, three and four printed transparencies. The colors are produced by using a stochastic distribution ${ }^{6,7}$ of the halftone dots to prevent moiré effects. They are printed on the 3M CG3460 transparency film with an inkjet printer, Canon Pixma Pro 9500 Mark II. By printing each ink at nominal surface coverages 0, 0.25, $0.5,0.75$ and 1 , one obtains 125 halftones. Each one is measured in reflectance and transmittance modes with the X-rite Color i7 instrument. The CIELAB $\triangle \mathrm{E} 94$ color distance enables assessing the prediction accuracy of the models. The color distance is obtained by converting the predicted and measured spectra into CIE-XYZ tristimulus values, calculated with a D65 illuminant and in respect to the $2^{\circ}$ standard observer, then by converting the CIE-XYZ values into CIELAB color coordinates using as a white reference the transmittance of the unprinted transparency deduced from the reflectance spectrum of the paper illuminated with the D65 illuminant ${ }^{11}$. In these experiments, all reflectances and transmittances are measured and predicted at normal incidence, i.e. $\theta_{0}=0$.

Regarding a single printed transparency, we tested the model in reflectance mode using Eq. (14) and in transmittance mode using Eq. (15). The results are given in Table 1 for both measurement modes. One can see that the average color distance is less than 0.4 for both predictions Note that the optimal $n$ value was limited to 10 when $n$ tends asymptotically to infinity.

Table 1. Prediction accuracy of a printed transparency in reflectance and transmittance modes.

\begin{tabular}{cccc}
\hline Measurement mode & Optimal $n$ value & Average $\Delta \mathrm{E}_{94}$ & 95\%-quantile \\
\hline Reflectance & 10 & 0.15 & 0.48 \\
Transmittance & 2 & 0.34 & 0.87 \\
\hline
\end{tabular}

Regarding the stacks of printed transparencies, the model was tested in transmittance mode from three sets of 25 halftones. Table 2 presents the prediction accuracy of the model for stacks of two, three and four transparencies. One can notice that the color distance is less than 0.9 , which proves a good accuracy of the model. The average $\Delta \mathrm{E} 94$ slightly increases when the number of superposed transparency increases. This is mainly due to assumptions made in the model which are not perfectly satisfied: the inks are assumed to be non-scattering whereas the yellow ink slightly diffuses the light in reflectance mode. Consequently the reflectance spectrum is under-predicted and the predictions are less accurate with a large number of transparencies.

Table 2. Prediction accuracy for stacks of $N$ transparencies in transmittance mode.

\begin{tabular}{ccc}
\hline Number of transparencies & Average $\Delta \mathrm{E}_{94}$ & $95 \%$-quantile \\
\hline 2 & 0.55 & 2.03 \\
3 & 0.69 & 1.36 \\
4 & 0.86 & 1.36 \\
\hline
\end{tabular}


The use of stochastic halftoning is mandatory to avoid moirés but not sufficient if the different halftones are generated with the same algorithm. The correlation between the ink dot positions in the different halftone should be as low as possible. Otherwise, in case of strong correlation, a Glass pattern may appear ${ }^{12}$. This is especially the case when all the halftones are generated according to the same stochastic screen: since the locations of the ink dots in the different halftones are the same, any slight rotation of one halftone generates a Glass pattern. This artifact may be simply prevented by rotating the stochastic halftone screen by 90 degrees from one halftone to another.

\section{COLOR MATCHING APPLICATION}

An original application of the model consists in creating one stack color in transmittance mode from various combinations of colors printed on the transparencies. All the halftones are arbitrarily chosen except one, whose surface coverages are determined as those which minimize the difference between the targeted color and the one corresponding to the stack transmittance spectrum predicted by the model. Since the model is calibrated at normal incidence, the color matching effect is expected to be effective at normal incidence. However, the experience show that the color matching remains visually satisfying when observed at up to $30^{\circ}$ to the normal.

Let us explain how the different color combinations are determined for a stack of $N$ transparencies, whose spectral transmittance $T_{\text {stack }}(\lambda)$ is the target spectral transmittance to reproduce by superposing the $N$ halftones. In order to make the explanation easier to understand, we assume that this target spectral transmittance $T_{\text {stack }}(\lambda)$ can be obtained by printing one transparency with the nominal ink surface coverages $c_{s}, m_{s}$ and $y_{s}$ and by superposing it to $N-1$ unprinted transparencies. The $N$ transparencies are labeled with numbers $i=1,2, \ldots N$ corresponding to the superposition order. Note that in transmittance mode, the superposition order has no influence on the stack transmittance. The ink surface coverages of the halftone printed on the transparency $i$ are denoted as $c_{i}, m_{i}$ and $y_{i}$.

We arbitrarily choose nominal surface coverages $c_{i}, m_{i}$ and $y_{i}$ for the $N-1$ first halftones. In a first approximation, we can assume that the superposed halftones absorb as much light as one halftone with nominal surface coverages $\sum_{1}^{N-1} c_{i}$, $\sum_{1}^{N-1} m_{i}$ and $\sum_{1}^{N-1} y_{i}$. The surfaces coverages should therefore satisfy the condition:

$$
\begin{aligned}
c_{1}+c_{2}+\ldots+c_{N-1} & <c_{s} \\
m_{1}+m_{2}+\ldots+m_{N-1} & <m_{s} \\
y_{1}+y_{2}+\ldots+y_{N-1} & <y_{s}
\end{aligned}
$$

Then, we want to determine the nominal surface coverages $c_{N}, m_{N}$ and $y_{N}$ of the $N$ th halftone which provide the closest stack transmittance spectrum to the targeted one. The spectral transmittance of the stack is predicted by using iteratively Eqs. (9) to (11) from the transparencies' spectral reflectances and transmittances predicted by Eq. (3), respectively Eq. (4), in respect to their respective ink surface coverages. We intuitively know that the optimal $c_{N}, m_{N}$ and $y_{N}$ are close to the following ones

$$
c_{N}=c_{s}^{-} \quad \sum_{\overline{1}}^{N-1} c_{i}, m_{\bar{N}} \quad m_{s}=\sum_{1}^{N-1} m_{i}, \text { and } y_{N} \quad y_{s} \quad \sum_{1}^{N-1} y_{i} .
$$

We then vary $c_{N}, m_{N}$ and $y_{N}$ by steps of 0.01 until the $\Delta \mathrm{E}_{94}$ color difference between the predicted and targeted transmittance spectra reach a minimum. In practice, we may consider that optimal $c_{N}, m_{N}$ and $y_{N}$ values are obtained when the $\Delta \mathrm{E}_{94}$ is smaller than 0.5. By comparing the predicted and targeted transmittance spectra in terms of color difference, we do not precisely search for the $c_{N}, m_{N}$ and $y_{N}$ values providing the best spectral matching but the best color matching: the stack transmittance spectrum may be different from the target spectrum, but the corresponding color is perceived as the closest metameric color to the target color in respect to the considered illuminant. In our case, we selected the D65 illuminant. In order to avoid any dependence on the illuminant, we could also consider the sum of squared differences between the predicted and targeted spectra, instead of their color difference. However, it may occur 
that a perfect spectral matching cannot be achieved and that the best $c_{N}, m_{N}$ and $y_{N}$ values obtained with this method do not provide the closest color to the target color.

Figure 3 shows three examples of color matching applications with respectively two, three and four superposed transparencies. The pictures, captured in the normal direction to the transparencies with a digital camera, show the individual transparencies as well as the resulting stacks illuminated from beside by a light table. The transparencies are located at a few centimeters of the table in order to prevent multiple reflections which are not taken into account by the model. One can see that the target color looks quite uniform over the 30 samples. The average $\Delta \mathrm{E}_{94}$ is less than 0.4 for two and four superposed transparencies, which is lower than the defined tolerance of 0.5 . The maximum $\Delta \mathrm{E}_{94}$ value is around 1 because the color distances between the stacked colors and the target color are inside a sphere of radius 0.5 (centered on the Lab coordinates of the target color). The maximum $\Delta \mathrm{E}_{94}$ between two colors is therefore 1 . In the case of three stacked transparencies, the average is slightly higher than the tolerance, and is mainly due to printing artifacts on a few colors. Excluding the four farthest colors from the target, the average becomes 0.5 . To be sure that the maximum color deviation between the farthest colors in the stack image is lower than the perceptible color distance $\Delta \mathrm{E}_{94}=1$, we could add a constraint when choosing the color combinations by checking the maximum color deviation between each color and each other color.

(c)

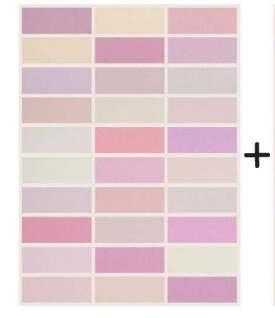

(a)
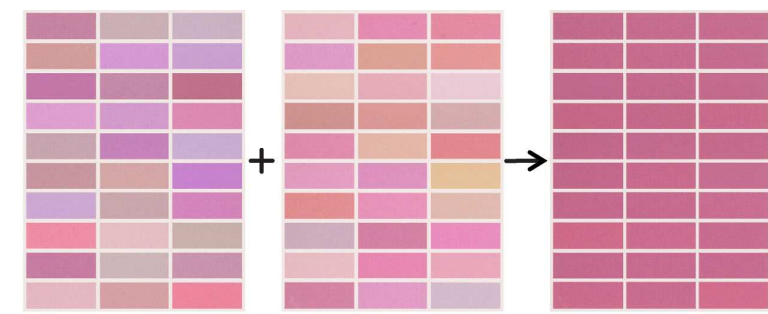

(b)
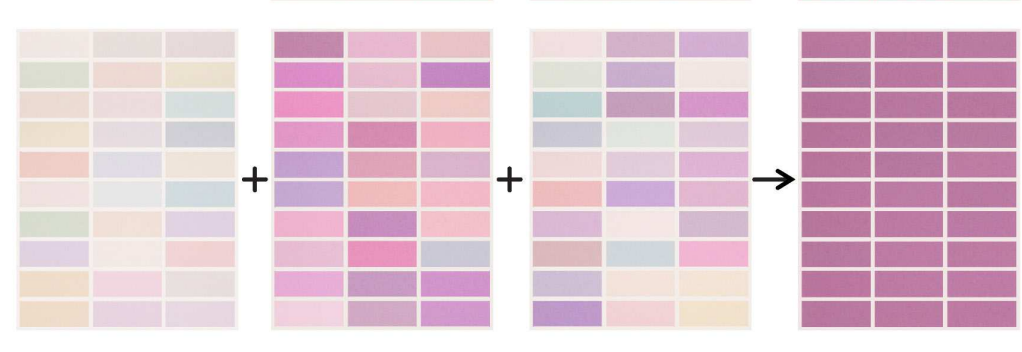

Average $\Delta \mathrm{E}_{94}: 0.58$

95\%-Quantile: 1.33

Maximum $\Delta \mathrm{E}_{94}$ distance: 1.87
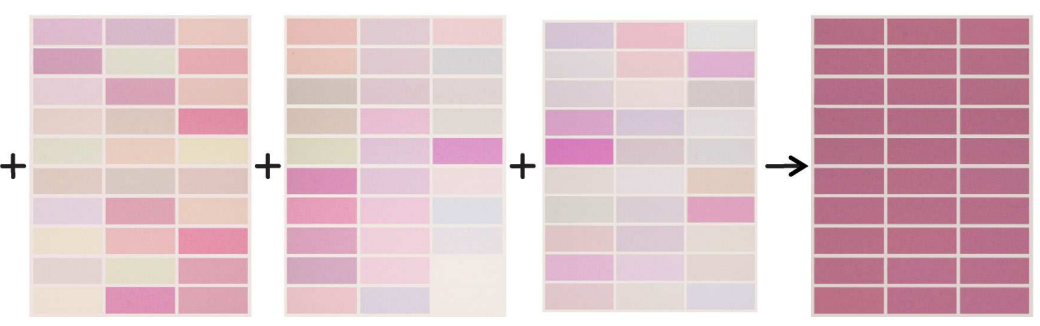

Average $\Delta \mathrm{E}_{94}: 0.29$

95\%-Quantile: 0.68

Maximum $\Delta \mathrm{E}_{94}$ distance: 0.92

Figure 3: Color matching application with (a) two, (b) three and (c) four transparencies. On the left side of the arrow, the individual transparencies are captured on the light table by a digital camera. On the right side of the arrow, the resulting stack is also illuminated by the light table.

Obviously, the set of possible combinations is wider if the targeted color is dark and unsaturated. For example, grey can be obtained by superposition of yellow and blue, or cyan and red, or magenta and green, and many other hue combinations, whereas a saturated red can only be obtained by superposition of yellow, red and magenta. 


\section{CONCLUSION}

In this work, we propose a spectral reflectance and transmittance model for stacks of transparencies printed in halftone. This model takes into account the multiple reflection processes occurring between the transparencies as well as the lateral propagation of the light between the halftone areas within each transparency using the Yule-Nielsen transform. The model's calibration only involves printing a few colors on one transparency and measuring their reflectance and transmittance spectra in the normal direction. The model then enables predicting the reflectance and transmittance spectra in any direction. Our experiment carried out on inkjet printed transparencies for observation in transmittance mode in the normal direction shows good prediction accuracy of the model. One may use the model to determine the exact ink surface coverages of halftones to print on the transparencies in order to obtain one target color for the stack. As several combinations of halftones can provide the targeted color, we can print several of them and assess visually the closeness of the obtained colors. The examples presented in this paper with two, three and four superposed transparencies, where thirty different color combinations are tested in each case, shows that the thirty obtained colors look satisfyingly similar and prove the accuracy of the prediction model. The main limitation of the model comes from the scattering of light by the inks. If the inks scatter too much, the prediction accuracy may decrease, all the more so as the number of transparencies is increased.

\section{REFERENCES}

[1] Hébert, M. and Hersch, R. D., "Yule-Nielsen based recto-verso color halftone transmittance prediction model," Appl. Opt. 50(4), 519-525 (2011).

[2] Hersch, R. D. and Crété, F., "Improving the Yule-Nielsen modified spectral Neugebauer model by dot surface coverages depending on the ink superposition conditions," Proc. SPIE 5667, 434-445 (2005).

[3] Kubelka, P., "New contributions to the optics of intensely light-scattering materials, part II: Non homogeneous layers, " J. Opt. Soc. Am. 44, 330-335 (1954).

[4] Hébert, M., Hersch, R. D. and Simonot, L., "Spectral prediction model for piles of nonscattering sheets," J. Opt. Soc. Am. A 25(8), 2066-2077 (2008).

[5] Amidror, I., [The theory of the moiré phenomenon: Periodic layers], 2nd ed., Springer, Berlin (2009).

[6] Ostromoukhov, V. and Hersch, R. D., "Stochastic clustered-dot dithering," J. Electronic Imaging 8, 439 (1999).

[7] Qi, M., Yang,C., Tu, C., Meng, X. and Sun, Y., "A GPU-based algorithm for building stochastic clustered-dot screens," Advances in Visual Computing 4841, 98-105 (2007).

[8] Stover, J., [Optical scattering: measurement and analysis], SPIE-International Society for Optical Engineering (1995).

[9] Born, M., Wolf, E. and Bhatia, A., [Principles of optics: electromagnetic theory of propagation, interference and diffraction of light], Cambridge Univ Pr. (1999).

[10] Amidror, I. and Hersch, R. D., "Neugebauer and Demichel: dependence and independence in $n$-screen superpositions for colour printing," Color Research and Application 25, 267-277 (2000).

[11] Sharma, G., [Digital Color Imaging Handbook], CRC Press, 30-36 (2003).

[12] Amidror, I., [The theory of the moiré phenomenon: Aperiodic layers], Springer, Berlin (2007). 